Ann. Génét. Sél. anim., 1974, 6 (3), 387-390.

\title{
UN CAS DE CHROMOSOME Y ANORMALEMENT LONG CHEZ BOS TAURUS L.
}

\author{
E. P. CRIBIU et C. P. POPESCU \\ avec la collaboration technique de Jeannine Boscher \\ U. N.C. E. I. A. et Laboratoire de Génétique factorielle, \\ Centre national de Recherches zootechniques, I. N. R. A., \\ 783.50 Jouy en Josas
}

RÉSUMÉ

Un chromosome $\mathrm{Y}$ anormalement long est décrit chez un mâle de race Charolaise phénotypiquement normal. Les longueurs relatives du chromosome $\mathrm{Y}$ entier et de chacun des deux bras sont significativement différentes de celles d'un Y normal. L'index centrométrique n'est pas modifié et les bandes $\mathrm{C}$ et $\mathrm{G}$ sont apparemment similaires à celles d'un Y normal. La taille exceptionnellement longue serait due à un degré différent de spiralisation de la chromatine.

Gustavsson et al. (I968) ont décrit la première anomalie de structure affectant un chromosome sexuel chez les bovins : une translocation $\mathrm{X} /$ autosome. Plus récemment, FECHHEIMER (I973) a trouvé chez un mâle de race Ayrshive un chromosome Y anormalement long.

Nous rapportons dans cette note un autre cas d'allongement du chromosome $\mathrm{Y}$, le deuxième $Y$ "géant " rencontré dans cette espèce.

L'étude porte sur un taureau Charolais âgé d'un an, phénotypiquement normal. Deux séries de cultures de sang ont été faites selon la technique de DE Grouchy et al. (I964) et les chromosomes de I 5 cellules ont été mesurés selon une méthode décrite antérieurement (CRIBIU, r974). Deux autres séries de lames ont été traitées pour les bandes C selon la méthode de Sumner (r972) et les bandes G d'après la méthode de SEABRICht (I97I).

Toutes les cellules étudiées contenaient le nombre de base normal $2 n=60$ dont 58 autosomes acrocentriques et deux chromosomes sexuels submétacentriques. Les longueurs relatives moyennes du bras court, du bras long et du chromosome Y entier obtenues sur les $I_{5}$ cellules mesurées ont été comparées avec celles d'un idiogramme de référence (CRIBIU et PoPESCU, i974). Le test $t$ indique des différences très hautement significatives au seuil de I p. I ooo pour toutes les données (tabl. I). Exprimé en pourcentages l'allongement du chromosome Y serait de l'ordre de $12,56 \mathrm{p}$. Ioo pour le bras court et de 20,22 p. Ioo pour le bras long. L'index centromérique moyen reste voisin de celui de l'Y normal de l'idiogramme de référence. 

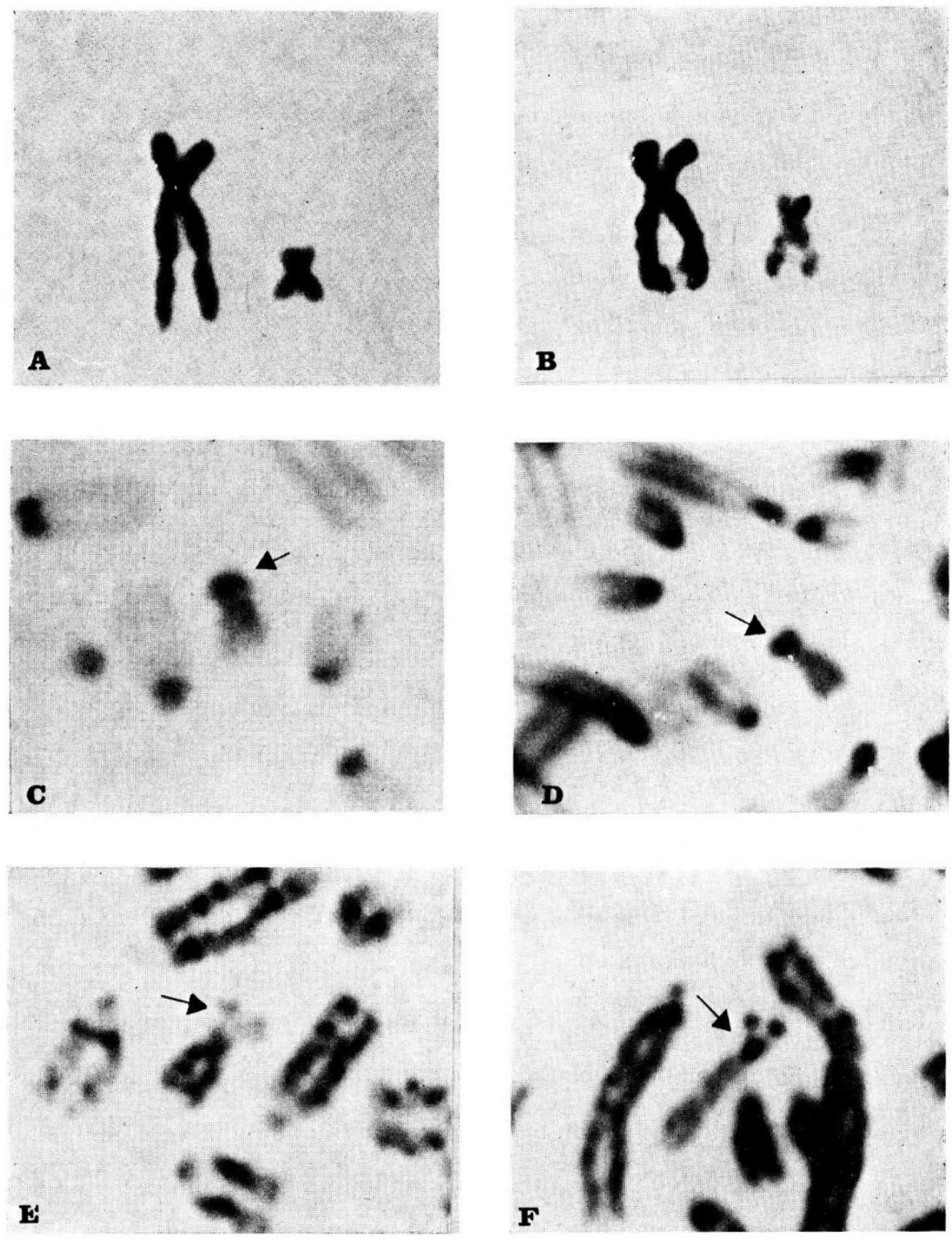

FIg. I. - Chromosomes sexuels normaux et $Y$ " allongé " chez un mâle Charolais

A : Chromosomes sexuels $\mathrm{X}$ et $\mathrm{Y}$ d'un mâle normal ;

$B$ : Chromosomes $X$ et $Y$ de l'animal porteur de I' $Y$ " géant ";

C : Fragment de métaphase normale traitée par la méthode des bandes C. Le chromosome $Y$ est marqué par une flèche ;

$\mathrm{D}$ : Les bandes $\mathrm{C}$ sur un chromosome $\mathrm{Y}$ " allongé „ (marqué par une flèche);

E : Fragment de métaphase normale traitée par la méthode des bandes G (l'Y est marqué par une flèche);

$\mathrm{F}$ : Les bandes $\mathrm{G}$ sur un chromosome $\mathrm{Y}$ " allongé " (marqué par une flèche). 
L'accroissement de la taille de l'Y qui implique les deux bras et conserve un index centromérique normal supposerait deux remaniements successifs : deux translocations sur chacun des bras ou une translocation suivie d'une inversion péricentrique.

\section{TABLEAU I}

Comparaison des longueurs moyennes de l' $Y$ "allongé " avec l'idiogramme de référence

\begin{tabular}{|c|c|c|c|c|c|}
\hline Chromosome Y & $\begin{array}{c}\text { Y " allongé " } \\
\text { moyenne sur } 15 \\
\text { cellules }\end{array}$ & $\begin{array}{l}\text { Idiogramme } \\
\text { de référence }\end{array}$ & $t$ & $\begin{array}{c}\text { Degrés } \\
\text { de liberté }\end{array}$ & Signification \\
\hline Longueur totale $\left(\mathrm{Y}_{t}\right)$ & 27,44 & 23,46 & 5,23 & 105 & $P<0,001$ \\
\hline Bras court $\left(Y_{p}\right)$ & 11,20 & 9,94 & 3,50 & 105 & $\mathrm{P}<0,001$ \\
\hline Bras long $\left(Y_{q}\right)$ & 16,23 & 13,50 & 5,07 & 105 & $P<0,001$ \\
\hline
\end{tabular}

Cette explication est peu probable et les images de l'Y " allongé " obtenues par les méthodes des bandes $\mathrm{C}$ et $\mathrm{G}$ sont similaires à celles d'un $\mathrm{Y}$ normal (fig. I). En effet, les blocs d'hétérochromatine constitutive, mis en évidence par la méthode des bandes $\mathrm{C}$ sur les bras courts, semblent identiques (fig. I C et D). Par la méthode des bandes G l'on observe, aussi bien sur 1'Y allongé (fig. I F) que sur l'Y normal (fig. I E), deux bandes sombres sur le bras court, une proximale et une autre distale par rapport au centromère. Entre elles il y a une région claire correspondant à l'emplacement de l'hétérochromatine constitutive qui, elle, ne se colore pas par cette technique. Sur les bras longs des deux $\mathrm{Y}$ on distingue trois bandes sombres.

Si l'hypothèse des remaniements est écartée, la taille anormalement grande de ce chromosome pourrait être expliquée par un degré différent de spiralisation de la chromatine. Cette possibilité a d'ailleurs été invoquée pour expliquer le polymorphisme de la taille du chromosome $Y$ chez les humains (Wennstrom et De la Chapelle, ig63).

Quoi qu'il en soit, une étude plus approfondie du polymorphisme de l'Y chez Bos taurus L. serait souhaitable, parce que les variations de sa taille pourrait en faire un marqueur génétique (GENest et al., r970).

Reçu pour publication en septembre $19 \pi 4$.

\section{SUMMARY}

\section{AN ABNORMALLY ELONGATED Y CHROMOSOME IN BOS TAURUS I.}

An elongated $\mathrm{Y}$ chromosome is described in a Chavolais bull with a normal phenotype. The mean of the relative length of entire $\mathrm{Y}$ chromosome is highly significantly different from this of normal $\mathrm{Y}$.

The centromeric index is normal and the $\mathrm{C}$ - and G-banding pattern are like a normal $\mathrm{Y}$. The lenght variation is probably due to the degree of spiralisation of the chromatid.

\section{RÉFÉRENCES BIBLIOGRAPHIQUES}

CRIBIU E. P., 1974. Observations sur les chromosomes somatiques chez Bos taurus $L$. Thèse de doctorat

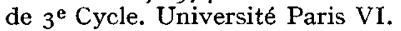

Cribiu E. P., Popescu C. P., 1974. Idiogramme de Bos taurus L. Ann. Génét. Sel. anim., 6 (sous presse). 
Fechieimer N. S., I973. A cytogenetic survey of young bulls in the U. S. A. Vet. Rec., 93, 535-536. Genest P., Laberge Cl., Poty J., Gagne R., Bouchard M., I97o. Transmission d 'un petit " $Y$ " durant onze générations dans une lignée familiale. Ann. Génét., 13, 233-238.

Grouchy J. (de), Rotbix P., Passage E., r964. Microtechnique pour l'étude des chromosomes humains à partir d'une culture de leucocytes sanguins. Ann. Génét. \%, 45.

Gustavsson I., Iraccaro M., Tiepolo L., Lindsten J., I968. Presumptive X-Autosome translocation in the cow. Preferential inactivation of the normal $X$ chromosome. Nature, 218, $183-184$.

Seabright II., I97I. A rapid Banding Technique for Human chromosomes. Lancet (773I), 97I-972.

Summer A. T., I972. A simple technique for demonstrating centromeric heterochromatin. Exp. Cell Res., 75, 304-306.

IVENNSTRON J., DF. i.a Chapelle A., I963. Elongation as the possible mechanism of origin of large human I" rhromosones. Hereditas, 50, 245-250. 